\title{
Optimal Evaluation of Suspected Choledocholithiasis: Does This Patient Really Have Choledocholithiasis?
}

\author{
Tae Yoon Lee \\ Division of Gastroenterology, Department of Internal Medicine, Konkuk University School of Medicine, Seoul, Korea
}

See "Sensitivity and Specificity of Magnetic Resonance Cholangiopancreatography versus Endoscopic Ultrasonography against Endoscopic Retrograde Cholangiopancreatography in Diagnosing Choledocholithiasis: The Indonesian Experience" by Dadang Makmun, Achmad Fauzi, Hamzah Shatri, on page 486-490.

Choledocholithiasis is a common biliary tract disease that causes numerous complications that can seriously influence the quality of life of patients. ${ }^{1}$ The diagnosis of choledocholithiasis is made on the basis of signs and symptoms, results of liver function test for cholestasis, and imaging findings. In patients with suspected choledocholithiasis, endoscopic retrograde cholangiopancreatography (ERCP) has been the gold standard for the diagnosis and treatment of choledocholithiasis in the past decades. The problem of ERCP is that it is a potentially invasive procedure with several complications such as post-ERCP pancreatitis, cholangitis, bleeding, and bowel perforation. ${ }^{2}$ Therefore, confirming the presence of choledocholithiasis before performing ERCP is frequently desirable. The reason this is important is that the risk-producing problems related to an unnecessary diagnostic ERCP increase if the patient does not have stones, while ERCP is beneficial for patients with bile duct stones. However, consensus about the optimal noninvasive diagnostic method for patients with suspected choledocholithiasis is lacking.

In this issue of Clinical Endoscopy, Makmun et al. ${ }^{3}$ conducted a retrospective study to compare sensitivity and specificity among

Received: September 6, 2017 Accepted: September 25, 2017

Correspondence: Tae Yoon Lee

Division of Gastroenterology, Department of Internal Medicine, Konkuk University School of Medicine, 120-1 Neungdong-ro, Gwangjin-gu, Seoul 05030, Korea Tel: +82-2-2030-7497, Fax: +82-2-2030-7458, E-mail: widebrow@empal.com

(cc) This is an Open Access article distributed under the terms of the Creative Commons Attribution Non-Commercial License (http://creativecommons.org/ licenses/by-nc/3.0) which permits unrestricted non-commercial use, distribution, and reproduction in any medium, provided the original work is properly cited. magnetic resonance cholangiopancreatography (MRCP), endoscopic ultrasonography (EUS), and ERCP for diagnosing choledocholithiasis. They came to the conclusion that EUS had better ability than MRCP to diagnose patients with true-positive and true-negative results for choledocholithiasis (sensitivity, $96 \%$ vs. $81 \%$ and specificity, $57 \%$ vs. $40 \%$ ). The positive predictive value (PPV) and negative predictive value (NPV) of EUS were also greater than those of MRCP (PPV, $85 \%$ vs. $74 \%$ and NPV, $80 \%$ vs. $50 \%$ ).

Several studies that compared EUS to MRCP showed both high diagnostic performance for choledocholithiasis, ${ }^{4}$ while a recent systematic review that included eight randomized trials demonstrated a slightly higher overall accuracy for EUS than for MRCP. ${ }^{5}$ This study also reported that EUS was superior to MRCP for detecting choledocholithiasis, which was confirmed using ERCP. However, I have a concern about the results of this study because the specificities of EUS and MRCP were much lower than those reported in previous studies. The authors did not explain clearly why the specificities of the two methods were low in this study. The most recent systematic review that included eight randomized, prospective, blinded trials revealed that the mean sensitivities of EUS and MRCP for detection of choledocholithiasis were $93.7 \%$ and $83.5 \%$, respectively, and their specificities were $88.5 \%$ and $91.5 \%$, respectively. ${ }^{5}$ In this study, a lower specificity means a high number of false-positive cases for both EUS and MRCP, which indicates that the ability of the examiner to diagnose choledocholithiasis by using the two modalities is not optimized and needs to be developed. A thickened common 
bile duct wall or pneumobilia might have been detected as a false stone image, therefore resulting in a false MRCP signal. ${ }^{6}$ To obtain a high sensitivity and specificity for MRCP, a high standard of technical expertise is important to ensure an accurate review of MRCP images, and this method requires proper patient cooperation. ${ }^{7}$ Regarding EUS, the presence of air bubbles inside the bile duct is a contributing factor to false-positive results. Despite its excellent visualization of the entire extrahepatic bile duct, the accuracy of EUS depends on subjective operator factors.

Deciding which test should be preferred first depends on several factors such as ease of availability, cost, patient-related factors, examination duration, and suspicion of a small stone. EUS is less invasive than ERCP, and MRCP is noninvasive. Therefore, the noninvasive nature of MRCP makes it a safer option for most patients, especially frail patients unfit for endoscopy. MRCP is a one-step evaluation that enables a comprehensive diagnostic assessment of the hepatobiliary system. ${ }^{1}$ However, MRCP may not be suitable for patients with claustrophobia, a cardiac pacemaker, a metal joint prosthesis, or a metal internal orthopedic fixation device. Moreover, the sensitivity of MRCP may be lower for small stones $(<6 \mathrm{~mm}),{ }^{8}$ and biliary sludge can be detected by EUS but generally not by MRCP. Conversely, the advantage of EUS is its superior ability to detect small stones, especially those in a nondilated common bile duct ${ }^{9}$ and its availability as an option for patients for whom MRCP is contraindicated (e.g., patients with a pacemaker). As a result, EUS should be considered for patients in whom the suspicion of choledocholithiasis remains moderate to high despite a negative MRCP. ${ }^{10}$ However, EUS may not be suitable for people with a surgically altered anatomy or gastrointestinal obstruction. Despite its overall high accuracy, the role of EUS in the diagnosis of common bile duct stones has not been firmly established because EUS is relatively more invasive than MRCP and does not permit therapeutic interventions. However, although EUS is a more invasive method than MRCP, it can be performed before ERCP during the same endoscopy session by the same endoscopist. ${ }^{11}$

To summarize, for patients with a high risk of choledocholithiasis (as determined by clinical presentation and noninvasive imaging), proceeding directly to ERCP is the best approach. ${ }^{12}$ For patients at low-to-moderate risk of choledocholithiasis, performing EUS or MRCP first before ERCP can prevent unnecessary ERCP. ${ }^{13}$ Owing to apparent pros and cons between these two modalities, the choice should be based on local availability and operator expertise. Further studies are needed to clarify the differences, which should include not only diagnostic performance but also the different sizes and types of stones, medical cost, and the potential for technical development, between EUS and MRCP for the diagnosis of choledocholithiasis. In my opinion, the best plan for investigating suspected common bile duct stones is to obtain an MRCP image first for patients without any contraindication. If the findings are negative but the clinical situation suggest otherwise, EUS should be performed to identify small stones or sludge that might have been missed by MRCP.

\section{Conflicts of Interest}

The author has no financial conflicts of interest.

\section{REFERENCES}

1. Pan S, Guo Q. Endoscopic ultrasonography versus magnetic resonance cholangiopancreatography for suspected choledocholithiasis: comments from the radiologists. Endosc Ultrasound 2016;5:129-131.

2. Freeman ML, Nelson DB, Sherman S, et al. Complications of endoscopic biliary sphincterotomy. N Engl J Med 1996;335:909-918.

3. Makmun D, Fauzi A, Shatri H. Sensitivity and specificity of magnetic resonance cholangiopancreatography versus endoscopic ultrasonography against endoscopic retrograde cholangiopancreatography in diagnosing choledocholithiasis: the Indonesian experience. Clin Endosc 2017;50:486-490.

4. Verma D, Kapadia A, Eisen GM, Adler DG. EUS vs MRCP for detection of choledocholithiasis. Gastrointest Endosc 2006;64:248-254.

5. De Castro VL, Moura EG, Chaves DM, Bernardo WM, Matuguma SE, Artifon EL. Endoscopic ultrasound versus magnetic resonance cholangiopancreatography in suspected choledocholithiasis: a systematic review. Endosc Ultrasound 2016;5:118-128.

6. Li P, Zhang Z, Li J, Jin L, Han W, Zhang J. Diagnostic value of magnetic resonance cholangiopancreatography for secondary common bile duct stones compared with laparoscopic trans-cystic common bile duct exploration. Med Sci Monit 2014;20:920-926.

7. Lee MG, Lee HJ, Kim MH, et al. Extrahepatic biliary diseases: 3D MR cholangiopancreatography compared with endoscopic retrograde cholangiopancreatography. Radiology 1997;202:663-669.

8. Zidi SH, Prat F, Le Guen O, et al. Use of magnetic resonance cholangiography in the diagnosis of choledocholithiasis: prospective comparison with a reference imaging method. Gut 1999;44:118-122.

9. Karakan T, Cindoruk M, Alagozlu H, Ergun M, Dumlu S, Unal S. EUS versus endoscopic retrograde cholangiography for patients with intermediate probability of bile duct stones: a prospective randomized trial. Gastrointest Endosc 2009;69:244-252.

10. Petrov MS, Savides TJ. Systematic review of endoscopic ultrasonography versus endoscopic retrograde cholangiopancreatography for suspected choledocholithiasis. Br J Surg 2009;96:967-974.

11. Unsal B, Alper E, Baydar B, et al. Combined use of endosonography and endoscopic retrograde cholangiopancreatography in the same session. Turk J Gastroenterol 2011;22:54-59.

12. Fogel EL, Sherman S. ERCP for gallstone pancreatitis. N Engl J Med 2014;370:1956

13. Sharma R, Menachery J, Choudhary NS, Kumar M, Puri R, Sud R. Routine endoscopic ultrasound in moderate and indeterminate risk patients of suspected choledocholithiasis to avoid unwarranted ERCP: a prospective randomized blinded study. Indian J Gastroenterol 2015;34:300304. 